Honic M., Kovacic I., Gilmutdinov I. and Wimmer M. (2020). "Scan to BIM for the semi-automated generation of a material passport for an existing building" In: Proc. 37th CIB W78 Information Technology for Construction Conference (CIB W78), São Paulo, Brazil, pp. 338-346. DOI: http://dx.doi.org/10.46421/2706-6568.37.2020.paper024

\title{
SCAN-TO-BIM FOR THE SEMI-AUTOMATED GENERATION OF A MATERIAL PASSPORT FOR AN EXISTING BUILDING
}

\author{
Meliha Honic ${ }^{1}$, Iva Kovacic ${ }^{2}$, Ildar Gilmutdinov ${ }^{3}$, and Michael Wimmer ${ }^{4}$
}

\begin{abstract}
The construction sector has a large negative impact on the environment due to its wasteful treatment of raw materials. To minimize the raw materials consumption, increasing recycling rates through reusing or recycling materials in existing stocks is of utmost importance. However, the main obstacle in the recycling of existing building stocks is the lack of information about materials incorporated in buildings.

This work presents the ongoing research within the multidisciplinary research project SCI_BIM, which received funding by the Austrian federal ministry of transport, innovation and technology. Within this paper, the semi-automated generation of an as-built BIM-Model for an existing building as well as the generation of a Material Passport (MP) is tested. In order to obtain the geometry and material information, laser scanners (geometry) and a Ground Penetrating Radar (GPR, for materials) are used. Thereby, first, a semi-automated BIM-Model is generated from the point cloud, which was obtained from the laser scans. Finally, the material information obtained from the GPR is integrated into the BIM-Model for the compilation of the MP.

First results show that the generation of the MP for the use case is possible, but confronted with manual steps, such as integrating material information from different sources (e.g. analysis of demolition experts). The results of this paper should serve as a basis for the creation of a secondary raw materials cadastre in order to enable recycling of valuable materials, thus supporting the achievement of the EU goals 20-20-20.
\end{abstract}

Keywords: Material Passport, Scan-to-BIM, Resources Efficiency, Urban Mining.

\section{INTRODUCTION}

The construction sector is responsible for $60 \%$ of all extracted raw materials (Bribián et al. 2011) as well as for $40 \%$ of energy-related CO2 emissions (Dean et al. 2016). There is urgent need for tools and methods for modelling and assessing the building stocks in order to generate a secondary raw materials cadastre, which could serve as basis for the realization of Urban Mining strategies. Urban Mining is promoting a systematic reuse of anthropogenic materials from urban areas, whereby in contrast to usual recycling, it

1 University Assistant, Institute of Interdisciplinary Construction Process Management, Vienna University of Technology, Vienna, Austria, Phone +43 (1) 58801 21543, meliha.honic@tuwien.ac.at

2 Professor, Institute of Interdisciplinary Construction Process Management, Vienna University of Technology, Vienna, Austria, Phone +43 (1) 58801 21526, iva.kovacic@tuwien.ac.at

3 Project Assistant, Institute of Visual Computing and Human-Centered Technology, Vienna University of Technology, Vienna, Austria, Phone +43 (1) 58801 18689, ildar.gilmutdinov@tuwien.ac.at

4 Professor, Institute of Visual Computing and Human-Centered Technology, Vienna University of Technology, Vienna, Austria, Phone +43 (1) 58801 18687, wimmer@cg.tuwien.ac.at 
includes, apart from mining of waste, also the exploration and observation of materials in buildings and infrastructures (Klinglmair and Fellner 2010). At present, there is a lack of knowledge on the material composition and construction of building stocks, which represents a major obstacle for realizing the Urban Mining strategy and therefore for increasing recycling rates (Brunner 2011).

For obtaining the geometry of buildings in the urban stock, in most cases laser scanning technology is used, which is already an established geometry acquisition method. In contrast to the geometry, for capturing the material composition, there is still no standardized method - which represents the main research gap: Currently there is no methodology for the comprehensive capturing and modelling of existing buildings including geometrical and material information and moreover for the semi-automated integration of materials information into a BIM-Model for the follow-up generation of a Material Passport (MP).

This paper builds up on the results of the research project SCI_BIM: Scanning and data capturing for Integrated Resources and Energy Assessment using Building Information Modelling as well as on the conference paper "BIM-supported scanning and data capturing for Integrated Resources and Energy Assessment" (Kovacic and Honic 2019), which was presented at the CIB World Building Congress 2019 in Hongkong.

The content of this paper is structured as follows. First, the state of the art regarding the scan-to-BIM process as well as a short introduction to Material Passports is provided. Second, the related research project SCI_BIM as well as the use case is described. Subsequently, the objectives and methodology are outlined. Finally, the results are presented followed by the conclusion.

\section{STATE OF THE ART}

\subsection{Scan-to-BIM}

In a typical Scan-to-BIM process, extensive human labor is required in order to generate a geometric model from an input point cloud, classify objects and establish their interrelations. There is a wide research on automation of this process, aimed to propose a framework that could at least partially reduce the need for human assistance.

While introducing semantic information into a BIM-model may require a professional engineer, geometry generation may be delegated to a computational process, as it is shown e.g. by Monszpart et al. (2015). They reconstruct an indoor scene by decomposing a scene into a set of planar primitives that constitute a regular arrangement. Namely, such arrangement, where primitives constitute of the user-specified angles between each other. Arikan et al. (2013) also generate a set of planar primitives, but is more flexible by allowing arbitrary polygons to describe a model shape. It also optimizes a model for certain criteria, but requires some human assistance to get an initial sketch of a building's geometry.

In contrast to previously mentioned works, Hong et al. (2015) and Jung et al. (2018) do not use planar reconstruction as the first step, but project the whole input cloud on a 2D plane. On a projected 2D point cloud, initial contouring is performed, followed by a regularization of contours segments. The advantage of this approach is the ability to analogously contour out other structural elements like walls and roofs, thus allowing the detection of openings like windows and doors. 


\subsection{Material Passport}

Material Passports (MP) have been defined variously by different parties. BAMB (Buildings as Material Banks) (n.d.), which is a Horizon 2020 project that has been finalized in 2019, describe MPs as electronic sets of data that provide the necessary information about materials, products and components for a circular use. The independent public platform Madaster describes MPs as a digital document that records the identity of all incorporated construction materials, by analysing the products and raw materials used in a building or project (Madaster n.d.).

Honic et al. (2019) developed a method for the compilation of a BIM-based MP, which documents the material composition of buildings. Thereby the MP serves as a planning and optimization tool in early design stages with regard to the efficient use of materials and subsequent demolition.

\section{RELATED RESEARCH PROJECT SCI_BIM}

This paper is based on the funded research project SCI_BIM: Scanning and data capturing for Integrated Resources and Energy Assessment using Building Information Modelling, which is conducted as collaboration of Faculties for Civil Engineering, Architecture, and Computer Science, as well as industrial partners - engineering and surveying companies 1,2 and 3 (C1, C2 and C3). The research project is conducted on a real use case, a facility of the university, which is described in 3.1. The aim of the project is to increase both resources as well as energy efficiency through coupling of technologies and methods for capturing and modelling (as-built BIM with geometry and material composition) of buildings and assets and finally using a gamification concept for the as-built model management by users.

\subsection{Use Case}

The use case (Fig. 1) is a lab and office facility of Vienna University of Technology with a total area of $1265 \mathrm{~m}^{2}$, located in the 3rd district of Vienna. The use case is a singlestorey building and comprises of 3 areas: office, lab and storage area. The three areas can also be distinguished from outside due to their varying heights. At the beginning of the research project the building was still occupied by its users, which made scanning a challenging task. The users of the facility moved out one year after the project start, which enabled a comprehensive data and information gathering through further scans. Since the building will be demolished medium-term, invasive methods on walls and slabs for obtaining detailed information about the materials and verification of the actual material composition could be applied.

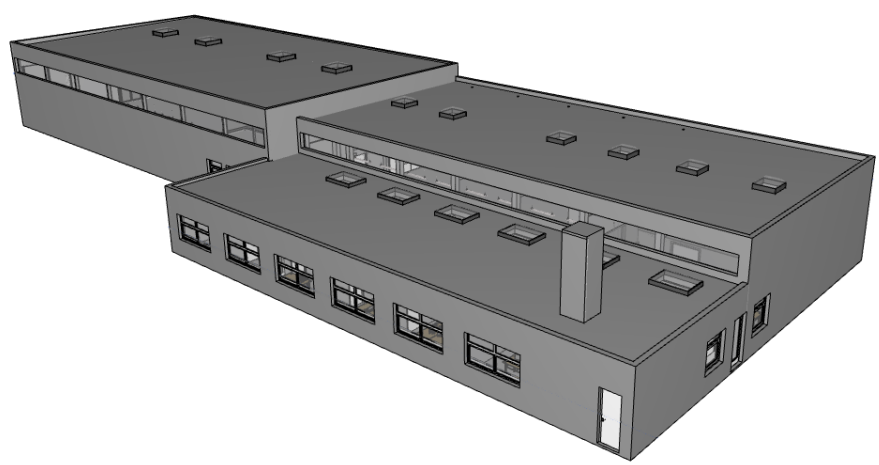

Figure 1: BIM-Modell generated by company 1. 


\section{OBJECTIVES}

The main objective of this paper is to build up a methodology for the semi-automated generation of a Material Passport for an existing building. Thereby the first step is to create a BIM-Model based on the point cloud obtained from laser scanning. The second step is to integrate the information about the incorporated materials into the BIM-Model, in order to generate an information rich BIM-Model which serves as basis for the MP. The final goal is the compilation of a MP for the existing building. The method for generating the MP is based on our previous research (Honic et al. 2019), where the MPmethod was used for assessing new buildings. In this paper, the existing MP-method will be optimized in order to make it applicable for existing buildings.

\section{METHODOLOGY}

\subsection{Data acquisition}

The building was scanned multiple times by three surveying companies (C1, C2 and C3 $=$ ZAMG Archeo Prospections), which all used different devices. C1 and C2 scanned the building in order to generate a geometry model, whereas C3 was responsible for obtaining materials information of the building. $\mathrm{C} 1$ used a high-tech handheld laser scanner, which generates a high resolution point cloud. C2 used a TLS (TLS = terrestrial laser scanner) with lower resolution of the produced point cloud. Two different devices were used in order to test their applicability and the quality of the point clouds. However, this paper focuses on the generation of an as-built BIM based on the point cloud obtained from the high-tech handheld scanner (C1), which is why the TLS is not further discussed in this paper. The material composition of the building is investigated through using a GPR (Ground Penetrating Radar). The data acquisition with GPR could not be conducted for all elements in one, since the building was occupied at the beginning of the data acquisition phase and therefore some of the elements could not be accessed. However, a comprehensive data acquisition will be possible after the final cleaning out of the building shortly before the demolition.

The GPR uses an electromagnetic wave as a signal carrier, which is radiated into the wall by a transmitting antenna. The electromagnetic wave diffuses in the wall with a material-dependent speed and is reflected at the interfaces of individual objects or from layers of different physical properties (permittivity, conductivity). The electromagnetic signal returning to the surface is captured by a receiver antenna and recorded digitally. The changes in the signal form (amplitude and frequency) allow conclusions about the physical properties of the irradiated components, such as their mineral composition, moisture, porosity, etc. The transit time of the signal is proportional to the distance of the reflecting interface. The electromagnetic energy radiated into the walls is damped differently depending on the component (material-related absorption loss). The decrease of the amplitude of the emitted energy momentum depends essentially on the conductivity of the component and the covered distance. The conductivity is the determining factor for the effective penetration depth of the electromagnetic waves. By comparing the amplitudes (transmitter, receiver) it is possible to differentiate the areas according to their absorption properties. Thus conclusions of different materials within the building parts are possible. However, the GPR can only detect different layers due to their different densities, but cannot determine the material compositions automatically. 
Therefore, an interpretation of the acquired data is necessary in order to make assumptions on the exact material composition of building elements.

\subsection{Points to BIM}

The two industrial partners C1 and C2 have generated two BIM-Models manually based on the obtained point cloud. However, a semi-automated creation of the as-built BIM was also conducted by partners of the university in order to test the automated as-built BIM generation as well as the integration of materials information into BIM. In order to generate a semi-automated BIM-Model out of the point cloud a computer-vision and image processing approach has been used. Thereby mainly the approach of Jung et al. (2018) is followed, where the core idea is detecting building objects from the projections of the cloud on a single plane. In order to obtain the heights of the three varying building parts, first a histogram that depicts the distribution of points along a certain height value, was generated. The four biggest peaks correspond to the floor and ceiling's heights of all three building parts. Based on the extracted height values, the point cloud was processed by creating sections for the points below a certain margin (current target height) and project them on a plane, thus a projection map was generated. The projection map was used for contouring in order to determine the outside contours of the building. The initial contour was detected by running the algorithm developed by Suzuki and Abe (1985) to find all closed contours on the image. Among the detected contours, the contour that included the largest floor area was selected. The resulting polyline consisted of waviness and had an excessive number of segments. In order to reduce the complexity of the polyline, curve decimation by Deuglas-Peucker algorithm was performed. After regularization of angles and detection of openings such as windows and doors through contouring, a BIM-Model was created by using the IFC format (Fig. 2). IfcOpenShell library was used which provides API between python code and IFC-file. Along the contours the exterior walls were created and its properties assigned according to the IFC Scheme. However, material properties of walls have not been assigned yet, which will be the next step, knowing that IfcOpenShell fully supports this functionality.

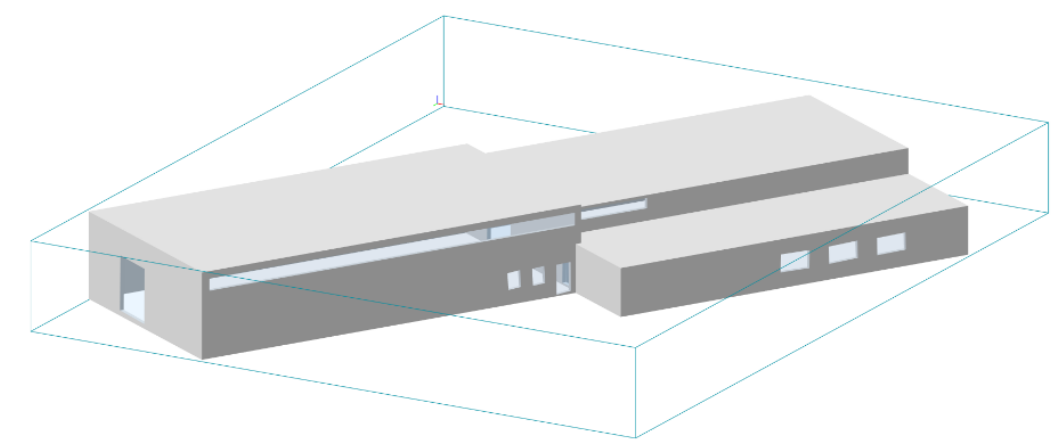

Figure 2: First result of the generated IFC-Model.

\subsection{Material Passport (MP) - method}

The applied MP-method is based on our previous research project BIMaterial: Process design for a BIM-based Material Passport, which was conducted at Vienna University of Technology. Within BIMaterial an MP-method for the assessment of the material composition, recycling potential etc. was developed for the preliminary and conceptual design stage, where the MP serves as optimization tool. The applied MP-method is based on the Austrian Institute for Building and Ecology (IBO n.d.) method, where each 
material is linked to a recycling grade from 1 to 5 . The recycling grades are obtained from the IBO-based tool "eco2soft" (n.d.). Grade 1 stands for $75 \%$ recycling and $25 \%$ waste and 5 for $0 \%$ recycling and for $125 \%$ waste. The additional $25 \%$ in case of grade 5 is due to auxiliary materials, which are required for demolishing a building. By linking the recycling grade of a specific material to the mass of that material, the share of recycling and waste in tons is assessed. In this paper, the MP-method is applied on a real use case for the first time, where the MP serves as documentation and inventory as well as shows the recycling potential of elements. As a comprehensive data acquisition has not been conducted yet, the MP-method for the end-of-life stage is demonstrated on an outside wall and not on the entire building. Data on the volumes are obtained from the BIMModel and the material composition from the GPR-measurements as well as from the contaminant investigation of a demolition expert.

\section{RESULTS}

Results show, that the semi-automated generation of the as-built BIM works properly, but needs further optimization. Since the building was scanned in occupied state, there is a lot of occlusion and noise that complicate the process of computer-vision procedures. Several objects became an obstacle between building and scanner, such as trees, which created holes in the point cloud. Furthermore, point clouds produce a large amount of data. In order to process the point cloud, a reduction of its size is required, sacrificing some details and precision. A further step will be the automated assignment of material information into the IFC-Model, through integration of data produced by the GPR-scans.

Since the produced IFC-model is not complete, the referential BIM-Model from C1 was used as basis for the compilation of the Material Passport. In the BIM-Model from $\mathrm{C} 1$ all building elements are classified via customized element IDs (e.g. AW-06 - exterior wall type 6). The material information of particular elements is coupled to the referential model via ID. 3 different wall classifications were identified: concrete (in dark grey: concrete wall type 1 and light grey: concrete wall type 2), brick (dark orange: brick wall type 1, light orange: brick wall type 2 and pink: brick wall type 3 ) as illustrated in Fig. 3. The MP has been created for exterior wall 06 (AW-06 in Fig. 3), thus for concrete wall type 1 . The detailed composition of the exterior wall 06 was obtained from assessments of the demolition expert. Three parts of exterior wall 06 were scanned with GPR, however there are 3 parts in between which were not scanned. For the MP-assessment it is assumed that the missing parts in between are also of the same type. The total area of the wall, which is $147,5 \mathrm{~m}^{2}$, is obtained from the BIM-Model. Due to its trapezoidal shape the area of the sheet has been estimated as $191,75 \mathrm{~m}^{2}$ ( $+30 \%$ of the actual area).

Table 1 shows the input parameters, which are required to obtain the mass of all materials within the wall. The materials as well as their density, surface weight and the thickness were obtained from the analysis of the demolition expert. In Table 2, the results of the MP-assessments are illustrated. The recycling grades are obtained from IBO and changed based on the analysis results of the demolition expert. The recycling grade of the trapezoidal sheet out of steel is usually 1, as proposed by IBO. However, it was detected that the steel sheet is not fully pure such that more effort for the recycling is required as well as loss of recyclable steel sheet will occur, which is why the recycling grade has been downgraded to 3 (25\% recycling and $75 \%$ waste). The insulation material is mineral wool. Results of the analysis have shown that the mineral wool contains asbestos, which is a hazardous waste and needs to be separated from the other materials. Therefore the recycling grade of mineral wool has been changed from the initial grade 3 
based on IBO to 5 ( $0 \%$ recycling and $125 \%$ waste). The aerated concrete is not contaminated and therefore the recycling grade 2 (50\% recycling 50\% waste) from IBO did not need to be changed. Results show that in total the exterior wall 06 leads to about 15 tons of recyclable mass and to 16 tons of waste mass. In order to obtain results for the entire building, each element will be assessed on element-level and finally summed up to the building-level.

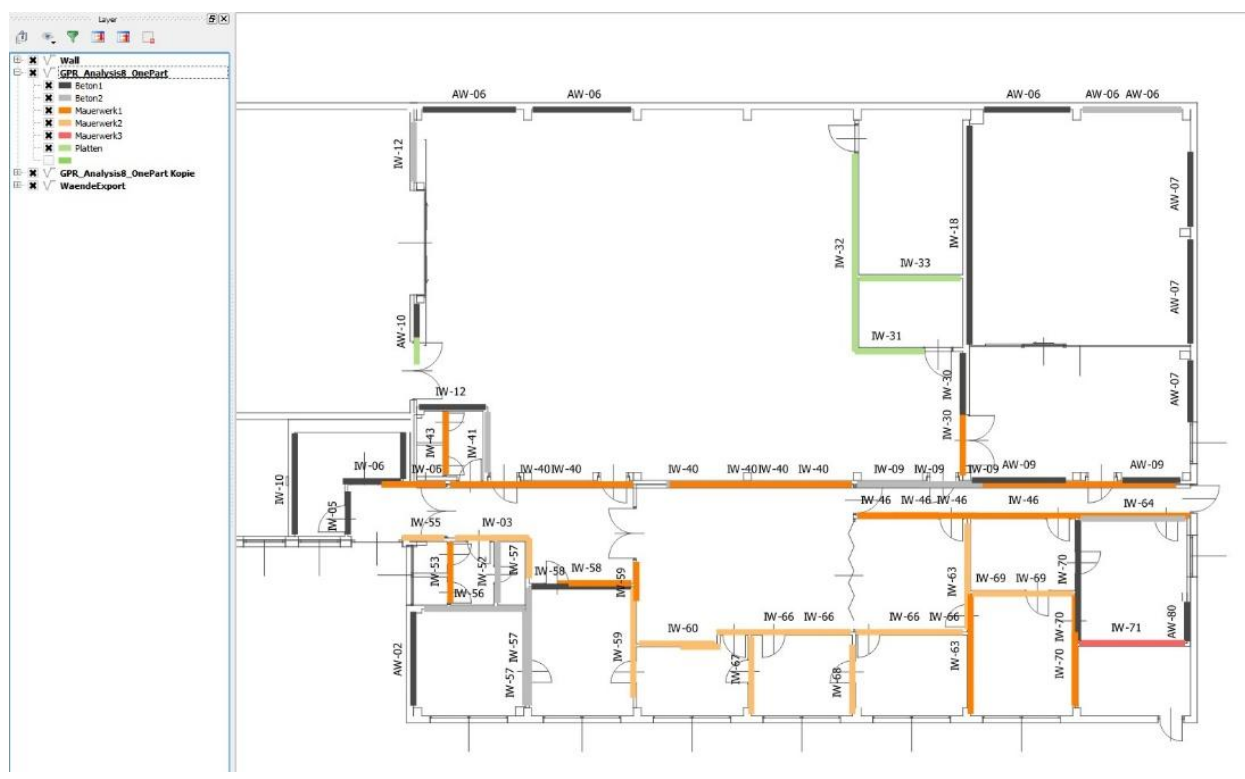

Figure 3: Material classification @ZAMG Archeo Prospections.

Table 1: Input parameters for the MP-assessments.

\begin{tabular}{lccccc}
\hline $\begin{array}{l}\text { exterior wall 06 } \\
(\mathrm{AW}-06)\end{array}$ & $\begin{array}{c}\text { density } \\
{\left[\mathrm{kg} / \mathrm{m}^{3}\right]}\end{array}$ & $\begin{array}{c}\text { surface weight } \\
{\left[\mathrm{kg} / \mathrm{m}^{2}\right]}\end{array}$ & $\begin{array}{c}\text { thickness } \\
{[\mathrm{m}]}\end{array}$ & $\begin{array}{c}\text { area } \\
{\left[\mathrm{m}^{2}\right]}\end{array}$ & $\begin{array}{c}\text { mass } \\
{[\mathrm{t}]}\end{array}$ \\
\hline $\begin{array}{l}\text { trapezoidal sheet } \\
\text { (steel) }\end{array}$ & 10 & & 191,75 & 1,92 \\
mineral wool & 15 & 0,05 & 147,50 & 0,11 \\
aerated concrete & 800 & 0,25 & 147,50 & 29,50 \\
\hline
\end{tabular}


Table 2: Results of the MP-assessments.

\begin{tabular}{lccccc}
\hline $\begin{array}{l}\text { exterior wall 06 } \\
\text { (AW-06) }\end{array}$ & $\begin{array}{c}\text { recycling grade } \\
\text { from IBO } \\
{[1-5]}\end{array}$ & $\begin{array}{c}\text { analysis of the } \\
\text { demolition } \\
\text { expert }\end{array}$ & $\begin{array}{c}\text { optimized } \\
\text { recycling } \\
\text { grade }\end{array}$ & $\begin{array}{c}\text { share of } \\
\text { recycling } \\
{[\mathrm{t}]}\end{array}$ & $\begin{array}{c}\text { share of } \\
\text { waste } \\
{[\mathrm{t}]}\end{array}$ \\
\hline $\begin{array}{l}\text { trapezoidal sheet } \\
\text { (steel) }\end{array}$ & 1 & low purity & 3 & 0,48 & 1,44 \\
mineral wool & 3 & $\begin{array}{c}\text { containing } \\
\text { asbestos }\end{array}$ & 5 & - & 0,14 \\
aerated concrete & 2 & 2 & 14,75 & 14,75 \\
\hline
\end{tabular}

\section{CONCLUSION}

In this paper, a methodology for generating a BIM-based Material Passport for an existing building was presented. The methodology is based on coupling of digital technologies and methods for scanning and modelling an as-built BIM of a use case for further assessment of the incorporated materials. For data acquisition laser scanning and GPR-technology was used, whereby from the laser scans the geometry and from the GPR the incorporated materials of the use case were obtained. On the one hand, the asbuilt BIM was created manually and on the other hand, a semi-automated approach was tested, which requires further work in order to generate a complete BIM-Model. Based on the BIM-Model and analysis of the demolition expert, a Material Passport was compiled for one exterior wall, demonstrating the MP-method.

Results show that the generation of a semi-automated as built-BIM is confronted with obstacles such as occlusion and noise that complicate the process of computervision procedures. Several objects such as trees were also included in the scans, which created holes in the point cloud. Furthermore, point clouds produce a large amount of data, which required a reduction of its size and therefore lead to some information loss. Therefore, the manually created BIM-Model from company 1 was used for the compilation of the MP. The results of the applied MP-method on the exterior wall of the use case produced valuable results by showing e.g. the total share of recycling and waste of materials. However, the methodology is confronted with manual steps such as integrating materials information from different sources, e.g. analysis through bores on the use case by a demolition expert.

Further steps will be the optimization of the as-built BIM generation as well as the automated integration of material information into the BIM-Model. Moreover, based on the information rich BIM-Model, an automated MP should be compiled by using the bidirectionally connected tool (BuildingOne n.d.). The tool serves as database, where information on e.g. recycling potential of materials is stored as well as a calculator of the MP-results. Through the toolchain BIM - BuildingOne (n.d.) changes in the BIM-Model are synchronized and results recalculated automatically.

Since at present, there is a lack of knowledge on the material composition and construction of building stocks, representing the major obstacle for the realization of the Urban Mining strategy and therefore for the increase of recycling rates, the developed methodology could serve as basis for the creation of a secondary raw materials cadastre on city-level. The methodology, consisting of integrated assessment of geometry and 
materials for follow-up generation of MPs, could support the achievement of the EU goals 20-20-20 by enabling the reuse and recycling of valuable materials.

\section{REFERENCES}

Arikan, M., Schwärzler, M., Flöry, S., Wimmer, M., and Maierhofer, S. (2013). O-snap: Optimization-based snapping for modeling architecture. ACM Transactions on Graphics (TOG), 32(1), pp. 1-15.

BAMB - Buildings as Material Banks (n.d.). Available at: https://www.bamb2020.eu/, [Accessed 29. Jan. 2020].

Bribián, I.Z., Capilla, A.V., and Usón, A.A. (2011). Life cycle assessment of building materials: Comparative analysis of energy and environmental impacts and evaluation of the eco-efficiency improvement potential. Building and Environment, 46(5), pp. 1133-1140.

Brunner, P. H. (2011). Urban mining a contribution to reindustrializing the city. Journal of Industrial Ecology, 15(3), pp. 339-341.

BuildingOne (n.d.). Available at: http://www.onetools.de/, [Accessed 29. Jan. 2020].

Dean, B., Dulac, J., Petrichenko, K., and Graham P. (2016). Towards zero-emission efficient and resilient Buildings. Global Status Report.

Eco2soft (n.d.). Available at: https://www.baubook.info/eco2soft/, [Accessed 29. Jan. 2020].

Hong, S., Jung, J., Kim, S., Cho, H., Lee, J., and Heo, J. (2015). Semi-automated approach to indoor mapping for 3D as-built building information modeling. Computers, Environment and Urban Systems, 51, pp. 34-46.

Honic, M., Kovacic, I., Sibenik, G., and Rechberger, H. (2019). Data- and stakeholder management framework for the implementation of BIM-based Material Passports. Journal of building engineering, 23, pp. 341-350.

IBO - Austrian Institute for Building and Ecology (Österreichisches Institut für Bauen und Ökologie $\mathrm{GmbH}$ ) (n.d.). Available at: https://www.ibo.at/, [Accessed 29. Jan. 2020].

Jung, J., Stachniss, C., Ju, S., and Heo, J. (2018). Automated 3D volumetric reconstruction of multiple-room building interiors for as-built BIM. Advanced Engineering Informatics, 38, pp. 811-825.

Klinglmair, M., and Fellner, J. (2010). Urban mining in times of raw material shortage: Copper management in Austria during World War I. Journal of Industrial Ecology, 14(4), pp. 666-679.

Kovacic, I., and Honic, M. (2019). BIM-supported scanning and data capturing for Integrated Resources and Energy Assessment. CIB WBC Conference 2019, Hongkong.

Madaster (n.d.). Available at: https://www.madaster.com/en, [Accessed 29. Jan. 2020].

Monszpart, A., Mellado, N., Brostow, G. J., and Mitra, N. J. (2015). RAPter: rebuilding man-made scenes with regular arrangements of planes. ACM Trans. Graph., 34(4), pp. 103-1.

Suzuki, S., and Abe, K. (1985). Topological structural analysis of digitized binary images by border following. Computer vision, graphics, and image processing, 30(1), pp. 3246. 